Comment

\title{
Some remarks on the time of flight and range of a projectile in a linear resisting medium
}

\author{
S. M. Stewart \\ Department of Mathematics, The Petroleum Institute, PO Box 2533, Abu Dhabi, United Arab Emirates.
}

Received 8 November 2009; Revised 13 July 2010; Accepted 6 February 2011

\begin{abstract}
In view of the recent work by Karkantzakos [Journal of Engineering Science and Technology Review 2 (2009) 76-81], a number of remarks highlighting the connection between the Lambert W function and the time of flight and range of a projectile moving in a resisting medium where the retarding force acting on the projectile is proportional to its velocity are made. In particular, we show how each of these quantities can be expressed in closed form in terms of the Lambert $\mathrm{W}$ function and indicate how the analysis of the motion becomes greatly simplified by its introduction.
\end{abstract}

Keywords: Linear resisted projectile motion, Lambert W function, time of flight, range.

\section{Introduction}

In a recent paper, Karkantzakos [1] presents an analysis of the classic problem of the motion of a projectile in air where the resistance is assumed to be proportional to its velocity. In this short comment we show how the time of flight and range for such a projectile can be written in closed form in terms of the now familiar Lambert $\mathrm{W}$ function $[2,3]$. When written in this way the problem of a projectile moving in a linear resisting medium becomes greatly simplified compared to that used by Karkantzakos since not only does it cast the solution in terms of a known function, but it also greatly facilitates in the analysis of its motion as all the standard tools of the calculus can be brought to bear on the function.

\section{Closed-form solutions}

Recall, for an object of mass $m$ launched with initial speed $\mathrm{u}_{0}$ from the origin at an angle of $\alpha$ to the horizontal $\left(0<\alpha<\frac{\pi}{2}\right)$ over level ground and in a gravitational field $g$ which is constant, in a linear resisting medium where the retarding force acting upon the object is considered to be proportional to its velocity, the wellknown equations of motion are

$$
\begin{aligned}
& x(t)=\frac{u_{0} \cos a}{\gamma}(1-\exp [(-\gamma t),]) \\
& y(t)=\left(\frac{g}{\gamma^{2}}+\frac{u_{0} \sin a}{\gamma}\right)(1-\exp [[(-\gamma t)]])-\frac{g t}{\gamma}
\end{aligned}
$$

Here $\gamma$ is a positive drag coefficient per unit mass and is assumed to remain constant during the motion of the projectile.

The time of flight $\mathrm{T}$ for the projectile is found by setting $y(T)=0$. On doing so, after rearranging terms algebraically, one finds

$$
u=\zeta\left(1-e^{-u}\right)
$$

Here $u=\gamma T, \zeta=1+c \sin \alpha$ while $c=\frac{\gamma v_{0}}{g}$.

Finding solutions to transcendental equations of the form given by Eq. (3) has occupied the time of many authors over the years [4-7]. Karkantzakos, like those before him, is the latest in a long line of such attempts. Traditionally, finding a closed-form solution for the time of flight in terms of known functions was not possible. Only recently with the emergence of the Lambert W function has such a closed-form solution become possible [8].

Rewriting Eq. (3) as, multiplying both sides by $e^{\zeta}$ and rearranging terms leads to

$$
(-\zeta+u) e^{-\zeta+u}=-\zeta e^{-\zeta}
$$

Since Eq. (4) is of the form of the defining equation for the Lambert $\mathrm{W}$ function (see the Appendix for a brief introduction to this function), its solution in terms of $\mathrm{W}$ is

$-\zeta(\gamma)+u=W_{0}\left(-\zeta(\gamma) e^{-\zeta(\gamma)}\right)$ 
$T(\gamma)=\frac{v_{0} \sin a}{g}\left(\frac{\zeta(\gamma)+W_{0}\left(-\zeta(\gamma) e^{-\zeta(\gamma)}\right)}{\zeta(\gamma)-1}\right)$

upon substituting for $\mathrm{u}$ and recasting into the zeta function form.

The selection of the principal branch is made on the following basis. Since the Lambert $\mathrm{W}$ function is the inverse of the function $f(x)=x e^{x}$, the following simplification rule for $\mathrm{W}$ holds

$x= \begin{cases}W_{0}\left(x e^{x}\right) & \text { for } \mathrm{x} \geq-1 \\ W_{-1}\left(x e^{x}\right) & \text { for } \mathrm{x} \leq-1\end{cases}$

Thus, since $\sin \alpha>0$ for all $\alpha \in(0, \pi / 2]$ and implies $\zeta>1$ as $\gamma>0$. Choosing the secondary real branch therefore results in $\mathrm{T}$ $=0$, a time corresponding to the instant the projectile is launched, and is easily seen as the trivial solution $(\mathrm{u}=0)$ of Eq. (3).

Karkantzakos' expression for the time of flight (see Eq. (12) in Ref. [1]) is given by

$$
T(\gamma)=-\gamma^{-1} b^{-1}(1+c \sin a)
$$

where $b^{-1}(x)$ is the inverse of the function $b(x)=\frac{x}{e^{x}-1}$ such that $x \in(-\infty, 0]$ (note the notation used in Eq. (8) has been changed to match the present work). Comparing our expression, namely Eq. (6), with Karkantzakos' we see his inverse b-function is nothing more than a thinly disguised Lambert $\mathrm{W}$ function. On unravelling its concealment one finds

$$
b^{-1}(x)=-x-W_{0}\left(-x e^{-x}\right)=-x+\sum_{n=1}^{\infty} \frac{n^{n-1}}{n !}\left(x e^{-x}\right)^{n}
$$

and is the inverse of Karkantzakos' b-function (see Eq. (41) in Ref. [1]). Note the series expansion for W given by Eq. (12) in the Appendix has been used. Substituting Eq. (9) for $b^{-1}(x)$ into Eq. (8) and noting $\gamma=\frac{g(\zeta-1)}{v_{0} \sin a}$, Eq. (6) immediately follows.

The advantage Eq. (6) has over the solution Karkantzakos presents stems from it being expressed in terms of a function now considered to be part of the standard canon of special functions. Karkantzakos' solution to the problem, while mathematically correct, suffers from being expressed in terms of an unfamiliar, nameless function. One may feel its introduction is more or less arbitrary. Motivated to solve the particular problem at hand, this tends to diminish its apparent appeal as its wider applicability beyond the linear resisted projectile motion problem goes unrecognised. Of course, this is exactly the reason why the Lambert W function for so long went unnoticed as many different authors, when faced with a similar problem, reverted to techniques not too dissimilar to those used by Karkantzakos. This need not be the case any longer.
Using the expression for the time of flight, the range readily follows. Substituting Eq. (6) for the time into Eq. (1) and recasting into a slightly simpler form using the defining equation for the Lambert W function yields

$R(\gamma)=\frac{v_{0}^{2} \sin 2 a}{2 g}\left(\frac{\zeta(\gamma)+W_{0}\left(-\zeta(\gamma) e^{-\zeta(\gamma)}\right)}{\zeta(\gamma)((\zeta)-1)}\right)$

as given by others [8-11].

\section{Conclusion}

We have shown how both the time of flight and range of a projectile in a linear resisting medium can be expressed in terms of the Lambert W function. The approach used by Karkantzakos, whose introduced b-function was designed to meet the particular mathematical demands of the problem at hand, suffers from requiring one to work with a function that not only lacks any form of familiarity, but whose most basic properties are not known in advance. While not being as widely known as it ought to be, the nascent nature of the Lambert $\mathrm{W}$ function should not detract from its use. Already a large number of applications for the Lambert W function have been found, and with greater awareness, this number will only grow.

\section{Appendix}

The Lambert $\mathrm{W}$ function, denoted by $W(z)$, is defined to be the inverse of the function $f(z)=z e^{z}$ satisfying

$$
W(z) e^{w(z)}=z
$$

Referred to as the defining equation for the Lambert W function, Eq. (11) has infinitely many solutions (most of which are complex) and is therefore multivalued. It is usual to write where $k=0, \pm 1, \pm 2, \ldots \ldots$ denotes the branch index for the function. When $\mathrm{Z}$ is real, only two branches of take on real values. By convention, the branch satisfying is taken to be the principal branch and is denoted by the branch satisfying is known as the secondary real branch and is denoted by.

If $\mathrm{X}$ is real, Eq. (11) can have either one unique positive real root if $x \geq 0$ except for; two negative real roots and if $-\frac{1}{e}<x<0$; one negative real root if $x=-\frac{1}{e}$; and no real roots if $x<-\frac{1}{e}$. The branch point between the two real branches occurs at $x=-\frac{1}{e}$.

A series expansion for the principal branch can be found using the Lagrange inversion theorem. The result is and converges if $|x|<\frac{1}{e}$, a result readily established by the ratio test. 


\section{References}

1. P. A. Karkantzakos, Time of flight and range of the motion of a projectile in a constant gravitational field under the influence of a retarding force proportional to the velocity, Journal of Engineering Science and Technology Review 2 (2009) 76-81.

2. R. M. Corless, G. H. Gonnet, D. E. G. Hare, D. J. Jeffrey, D. E. Knuth, On the Lambert W function, Advances in Computational Mathematics 5 (1996) 329-359.

3. P. B. Brito, F. Fabião, A. Staubyn, Euler, Lambert, and the Lambert W function today, Mathematical Scientist 33 (2008) 127-133.

4. N. D. Hayes, Roots of the transcendental equation associated with a certain difference-differential equation, Journal of the London Mathematical Society 25 (1950) 226-232.

5. E. M. Wright, Solution of the equation $z e^{z}=a$, Proceedings of the Royal Society of Edinburgh A 65 (1959) 193-203.

6. F. N. Fritsch, R. E. Shafer, W. P. Crowley, Algorithm 443: Solution of the transcendental equation $w e^{w}=x$, Communications of the ACM 16 (1973) 123-124.

7. C. E. Siewert, E. E. Burniston, Exact analytical solutions of $z e^{z}=a$, Journal of Mathematical Analysis and Applications 73 (1973) 626-632.

8. S. M. Stewart, An analytic approach to projectile motion in a linear resisting medium, International Journal of Mathematical Education in Science and Technology 37 (2006) 411-431.

9. R. D. H. Warburton, J. Wang, Analysis of asymptotic projectile motion with air resistance using the Lambert W function American Journal of Physics 72 (2004) 1404-1407.

10. E. W. Packel, D. S. Yuen, Projectile motion with resistance and the Lambert W function, The College Mathematics Journal 35 (2004) 337-350.

11. D. A. Morales, Exact expressions for the range and optimal angle of a projectile with linear drag, Canadian Journal of Physics 83 (2005) 67-83. 